\title{
Anti-adipogenic effects of viscothionin in 3T3-L1 adipocytes and high fat diet induced obesity mice
}

\author{
Sokho Kim', Seo-Hyun Ahn', Jong-Heum Park ${ }^{2}$, Chan Hum Park ${ }^{3}$, Yu Su Sin ${ }^{3}$, Gee-Wook Shin ${ }^{\text {* }}$ \\ and Jungkee Kwon ${ }^{1 *}$
}

\begin{abstract}
Viscum album subsp. Coloratum, also known as Korean mistletoe, is a traditional herb that has more recently been used for the treatment of nervine, hypertensive and cardiovascular diseases. Therefore, this study was undertaken to access the anti-obesity effect of Korean mistletoe-derived polypeptide viscothionin using 3T3-L1 adipocytes in vitro and in vivo mouse experimental model. Viscothionin (up to $5 \mu \mathrm{M}$ ) was used to treat mouse 3T3-L1 pre-adipocytes during adipocyte differentiation. Adipocyte differentiation in 3T3-L1 cells was confirmed by Oil Red O staining. Obesity was induced by a high-fat diet (HFD) in C57BL/6J mice, followed by oral administration of viscothionin (up to $10 \mathrm{mg} / \mathrm{kg}$ ) for 3 weeks. As a result, viscothionin ( $5 \mu \mathrm{M}$ ) inhibited differentiation of adipocyte cells and attenuated accumulation of intracellular lipids through activation of $5^{\prime}$-adenosine monophosphate-activated protein kinase (AMPK), by down-regulating phosphorylation in AKT and glycogen synthase kinase $3 \beta$ (GSK3 $\beta$ ). Treatment of viscothionin also decreased the levels of sterol regulatory element binding protein-1 (SREBP-1) and its target gene, fatty acid synthase (FAS). Moreover, viscothionin (10 mg/kg) significantly suppressed body weight and fat content, and improved serum lipid concentration, compared with the standard drug simvastatin $(10 \mathrm{mg} / \mathrm{kg})$, a well-known anti-obesity agent. The present study suggests, that viscothionin exerts anti-adipogenic effect through the activation of AMPK and has potential to prevent HFD-induced obesity.
\end{abstract}

Keywords: Viscum album, Viscothionin, Obesity, AMPK, In vivo mouse model

\section{Introduction}

Obesity is a complex chronic disorder which is linked to a number of health complications, including diabetes, hypertension, cancer, hyperlipidemia, osteoarthritis, and heart diseases [1]. 3T3-L1 cells are entrenched in vitro model for assessing the differentiation of adipocytes at a number of developmental stages, including, differentiation stages associated with obesity [2]. 3T3-L1 cells have been extensively studied for determining the anti-adipogenic effects of several existing and new therapeutic compounds that have potential to control body weight gain or

\footnotetext{
*Correspondence: shingw@jbnu.ac.kr; jkwon@jbnu.ac.kr

${ }^{1}$ Bio-Safety Research Institute and College of Veterinary Medicine,

Chonbuk National University, Iksan 54596, South Korea

Full list of author information is available at the end of the article
}

obesity. Thus suggesting that, the establishment of animal models could be useful to assess the anti-obesity potential of any therapeutic agents for the treatment of obesity. Studies have confirmed that mice fed with high-fat diet (HFD) are excellent obesity models in which the dietary conditions are the major contributors [3].

5 -Adenosine monophosphate-activated protein kinase (AMPK) is a heterotrimeric protein complex that acts as an energy sensor in homeostasis [4]. The activity of AMPK is achieved by the phosphorylation of critically self-contained threonine residue (Thr172) [5]. Phosphorylation of AMPK improves energy production by reducing the energy consumption of each biosynthetic pathway and increasing fatty acid oxidation [6]. AMPK acts in the regulation of glucose and adipocyte-associated lipid 
homeostasis, thus causes several metabolic changes [7]. Therefore, AMPK is known to be a significant target of anti-adipogenic and anti-obesity agents.

Mistletoe, a semi-parasitic plant, grows well on various types of trees worldwide. Mistletoe has several pharmacological activities, and can act as a nervine, hypertensive, vasodilator, or relaxant [8-10]. Korean mistletoe (Viscum album subsp. Coloratum) belonging to family Santalaceae, has been used as an herbal medicine that has recently been known to produce several bioactive components, including viscothionin [11]. However, no studies have been reported so far on viscothionin whether it possesses anti-adipogenic and anti-obesity effects along with an underlying mechanism for the observed activities. Therefore, herein this research, we investigated whether viscothionin activates AMPK, whose activation known to suppress adipogenesis. Consequently, we also performed in vivo studies in order to validate the anti-obesity effects of viscothionin using HFD-fed mice as an animal model.

\section{Materials and methods Chemicals}

Viscothionin isolated from Korean mistletoe was prepared with a slight modification of a previously reported method, and was used with purity over 97\% [12]. The insulin, dexamethasone (purity over 97\%), 3-isobutyl1-methylxanthine (purity over 99\%), dimethyl sulfoxide (DMSO), and compound C (AMPK inhibitor) were procured from Sigma (St. Louis, MO, USA). Simvastatin as a standard anti-obesity drug was purchased from Sigma (St. Louis, MO, USA). A kit from Oil Red O staining was purchased from Lifeline Cell Technology (Carlsbad, CA, USA). Primary antibodies for AMPK, GSK3 $\beta, p$-ACC, p-AKT, p-GSK3 $\beta, A C C, A K T$, phosphorylate (p)-AMPK, and $\beta$-actin were procured from Cell Signaling (Danvers, MA, USA). Anti-rabbit, or anti-mouse IgG secondary antibodies were purchased from Millipore (Bedford, MA, USA). Other ingredients and cell culture medium were obtained from Hyclone Ltd., Logan, UT, USA.

\section{Cell culture and adipocyte differentiation}

Mouse 3T3-L1 pre-adipocytes (American Type Culture Collection, Manassas, VA, USA) were cultured at $37{ }^{\circ} \mathrm{C}, 5 \% \mathrm{CO}_{2}$ atmosphere in Dulbecco's modified Eagle's medium (Gibco BRL, NY, USA) supplemented with $10 \%$ FBS and penicillin/streptomycin (Gibco BRL). The 3T3-L1 pre-adipocytes were incubated for 2 days until confluence. Here the cells were exposed to an adipogenic differentiation medium (DMI; DMEM containing 5\% FBS, $0.5 \mathrm{mM}$ 3-isobutyl-1-methylxanthine, $1 \mathrm{mM}$ dexamethasone, and $10 \mu \mathrm{g} / \mathrm{mL}$ insulin) for 4 days. After 2 days, the DMI was changed with DMEM containing $5 \%$ FBS and $10 \mu \mathrm{g} / \mathrm{mL}$ insulin, and then changed with
DMEM containing 5\% FBS every other day. The viscothionin was dissolved in phosphate buffered saline (PBS) at $100 \mathrm{mM}$, and then reconstituted in each medium at the working concentration. Additionally, cells were pretreated with compound $\mathrm{C}(20 \mu \mathrm{M})$ for one hour, then treated with viscothionin $(5 \mu \mathrm{M})$.

\section{Oil Red O staining}

Oil Red O staining was performed using the staining kit protocol described elsewhere. Oil Red O stained cultures were photographed using an Observer A1 microscope at magnification 100× (Carl Zeiss, Jena, Germany). After taking the photographs, the Oil Red $\mathrm{O}$ stained culture dye retained in the cells was eluted with isopropanol and quantified using spectrophotometer (absorbance at $540 \mathrm{~nm}$, PowerWave 2, Bio-Tek Instruments, Winooski, VT, USA).

\section{Real-time (RT)-PCR}

For RT-PCR analysis, A Mastercycler Gradient 5331 Thermal Cycler (Eppendorf, Germany) was used for the amplification of cDNA. Fluorescence signals were measured after each cycle for RT-PCR analysis using an ABI Step One Plus Sequence Detection System (Applied Biosystems, Branchburg, NJ, USA). A Primer Express Software (Applied Biosystems) was employed for the designing of specific sense and antisense RT-PCR primers which were as follows: forward $5^{\prime}$-GAAGTGGTG GAGAGACGCTTAC- $3^{\prime}$ and reverse $5^{\prime}$-TATCCTCAA AGGGCTGGACTG-3' for SREBP-1; forward $5^{\prime}$-CCC AGCCCATAAGAGTTACA- $3^{\prime}$ and 5'-ATCGGGAAG TCAGCACAA-3' for FAS; forward 5'-GCATGGCCT TCCGTGTTC- $3^{\prime}$ and reverse 5'- GATGTCATCATA CTTGGCAGGTTT- $3^{\prime}$ for GAPDH, housekeeping gene.

\section{Determination of intracellular triglyceride}

Intracellular triglyceride content was determined using a commercial triglyceride assay kit system (BioAssay, Hayward, CA, USA). Aliquots of various concentrations of viscothionin, with or without compound $C$ were used for the treatment of adipocyte cells in a 12-well plate during their differentiation. Then, the cells were washed with PBS and the cell suspension was homogenized after the addition of homogenizing solution $(154 \mathrm{mM} \mathrm{KCl}, 1 \mathrm{mM}$ EDTA, $50 \mathrm{mM}$ Tris, $\mathrm{pH}$ 7.4) followed by centrifugation (3000 rpm/5 min) of cell lysate for the removal of fat layer. The supernatants were then assessed for triglyceride content. Triglyceride content was normalized using the bovine serum albumin (BSA) as a standard.

\section{Immunoblotting analysis}

Proteins originated from the cell lysates were subjected to SDS-PAGE using $8 \%, 10 \%$ and $14 \%$ gel, and then 
transferred to polyvinylidene difluoride (PVDF) membranes (Bio-Rad, Laboratories, Hercules, CA, USA). 5\% skim milk was used for the blocking of the membranes, followed by incubation with each primary antibody in $1 \%$ skim milk at $4{ }^{\circ} \mathrm{C}$ overnight. Then, the blots were incubated with each secondary antibody for one hour, and signals were detected using a SuperSignal West Dura (Thermo Scientific, CA, USA). A ChemiImager (Alpha Innotech, San Leandro, CA, USA) was employed for analyzing the results.

\section{Animals and in vivo experimental design}

Forty male C57BL/6J mice, about 6 weeks old, were purchased from Dae-Han Biolink (Daejeon, Korea) and were maintained under pathogen-free conditions on a 12-h light/dark. The mice were randomly divided into two groups and were fed either a normal-diet $(n=8)$ or an HFD $(n=32)$ with $45 \%$ of the calorie fat. The HFD mice were fed for 6-week in order to induce obesity. After 6-week, the HFD-fed mice were randomly divided into four sub-groups and each sub-group was orally administered either $0.1 \mathrm{~mL}$ saline $(\mathrm{n}=8), 1 \mathrm{mg} / \mathrm{kg}$ viscothionin in $0.1 \mathrm{~mL}$ saline $(\mathrm{n}=8), 10 \mathrm{mg} / \mathrm{kg}$ viscothionin in $0.1 \mathrm{~mL}$ saline $(\mathrm{n}=8)$, or $10 \mathrm{mg} / \mathrm{kg}$ simvastatin (Sigma; purity over $97 \%$ ) in $0.1 \mathrm{~mL}$ saline daily for 3 weeks. The mice had free access to food and water and their food consumption was measured daily, while their weight gain was measured weekly. The weight gain/feed ratio was calculated as weight gain divided by total food intake. After fasting for $12 \mathrm{~h}$ and before collecting blood, all mice were scanned using micro-CT for abdominal visceral fat analysis. All mice were maintained according to the Chonbuk National University Institutional Guidelines for the care and use of experimental animals (Approval number: CBU 2018-0070; Approval date: November 28, 2018).

\section{Serum analyses}

All biochemical tests for obesity (triglyceride and total cholesterol concentrations) were performed on a VetTest 8008 Chemistry Analyzer from IDEXX, Seoul, South Korea. Levels of high-density lipoprotein (HDL) and lowdensity lipoprotein (LDL) were determined according to the instructions of kit manufacturer (Cell Biolabs, San Diego, CA, USA).

\section{Abdominal visceral fat analyses}

The mouse visceral fat distribution in the abdominal cavity was determined by micro-CT scanner (Skyscan-1072, TomoNT, Aartselaar, Belgium) between frames at a $10 \mu \mathrm{m}$ slice thickness. The visceral fat distribution was determined using dedicated software (CTAn v.1.8.1.2).

\section{Statistical analyses}

All the results are presented as mean $\pm \mathrm{SE}$, and analyzed by the Student's $t$-test, followed by one-way ANOVA analysis and Tukey's test. Values were considered significant at $p<0.05$.

\section{Results \\ Effects of viscothionin on AMPK activation}

To inquire whether the inhibition of adipocyte differentiation by viscothionin is mediated by AMPK activation, 3T3-L1 cells were treated by $5 \mu \mathrm{M}$ viscothionin for 8 days. Consistent with the increase in AMPK phosphorylation, the phosphorylation of acetyl-CoA carboxylase (ACC), a characterized phosphorylation target of AMPK, was also increased (Fig. 1b, c). However, AMPK phosphorylation inhibited the AKT activation and counteracted the glycogen synthase kinase $3 \beta$ (GSK3 $\beta$ ) inactivation (Fig. 1e). As shown in Fig. 1, viscothionin increased the phosphorylation of AMPK and ACC, while decreased the phosphorylation of AKT and GSK3 $\beta$, suggesting that viscothionin activated AMPK, then inactivated AKT and activated GSK3 $\beta$ to inhibit adipocyte differentiation in 3T3-L1 cells during differentiation. To confirm the effect of AMPK activation by viscothionin, 3T3-L1 cells were treated with compound $\mathrm{C}$, a pharmacological AMPK inhibitor. When the 3T3L1 cells were pre-treated with compound C $(20 \mu \mathrm{M})$, viscothionin-induced phosphorylation of AMPK and ACC were significantly reduced and phosphorylation of AKT and GSK3 $\beta$ was significantly increased (Fig. 1). These results indicate that viscothionin inhibited adipogenesis through AMPK activation.

\section{Effects of viscothionin on adipocyte differentiation involved in AMPK activation}

To clarify the effects of viscothionin on adipocyte differentiation involved in AMPK activation, we examined differentiation of viscothionin treated 3T3-L1 cells pre-treated with compound C. As shown in Fig. 2a, compound $\mathrm{C}$ significantly counteracted with inhibitory effect of viscothionin on adipocyte differentiation. Moreover, the effect on intracellular triglyceride levels in viscothionin-treated cells pre-treated with compound $\mathrm{C}$ was clearly counteracted as compared with viscothionintreated cells (Fig. 2b). Viscothionin-treated 3T3-L1 cells pre-treated with compound $C$ exhibited increased level of expression of sterol regulatory element binding protein-1 (SREBP-1) and fatty acid synthase (FAS), compared with viscothionin-treated cells (Fig. 2c, d). These results suggest that viscothionin-induced inhibition of adipogenesis in 3T3-L1 cells via AMPK activation. 

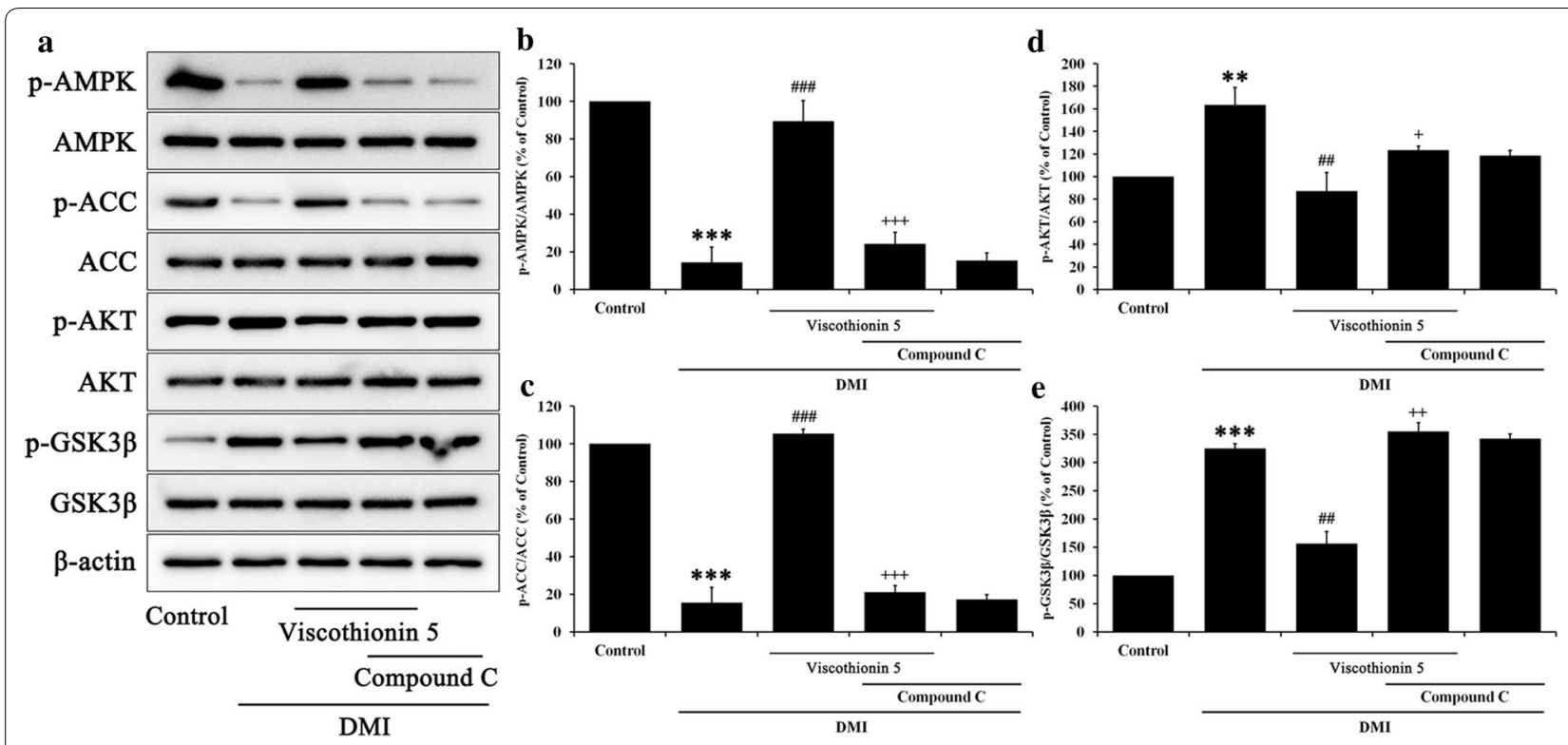

Fig. 1 Effects of viscothionin on AMPK signal pathway in 3T3-L1 cells. Cells were pre-treated with compound C for $1 \mathrm{~h}$, and then treated with $5 \mu \mathrm{M}$ viscothionin for 8 days. On day 8 , completely differentiated cells were lysed to extract total protein. a Protein extracts were prepared and subjected to Western blot analysis using p-AMPK, AMPK, p-ACC, ACC, p-AKT, AKT, p-GSK3 $\beta$, GSK3 $\beta$, and $\beta$-actin antibodies. Each total protein level was used as an internal control to elevate relative expression of the phosphorylated protein. $\mathbf{b}-\mathbf{e}$ Quantitative analysis of Western blot in $\mathbf{a}$. Data are represented as mean \pm SEM of three different preparations. ${ }^{* *} p<0.01$, and ${ }^{* * *} p<0.001$, as compared with control. ${ }^{\# \#} p<0.01$, and ${ }^{\# \#} p<0.001$, as compared with only DMI. ${ }^{+} p<0.05,{ }^{++} p<0.01$, and ${ }^{+++} p<0.001$, as compared with $\mathrm{DMl}+$ viscothionin $5 \mu \mathrm{M}$

\section{Effect of viscothionin on food intake and bodyweight}

Based on in vitro results, we performed in vivo experiments using HFD-induced obese mice. All the experimental mice showed no any symptoms of pathological condition or abnormalities during the experimental period. No significant differences among the groups in food consumption, except in control group (Fig. 3a) were observed. All groups had similar body weights at the beginning of the study $(22.7 \pm 1.1 \mathrm{~g})$, but the HFD mice experienced significant weight gain $(42.1 \pm 1.8 \mathrm{~g})$ compared with animals fed with the normal-diet $(32.7 \pm 1.7 \mathrm{~g})$ throughout the study. The HFD groups treated with viscothionin at the dose of $1 \mathrm{mg} /$ $\mathrm{kg}$ or $10 \mathrm{mg} / \mathrm{kg}$ gained less body weight $(40.5 \pm 1.5 \mathrm{~g}$; $35.3 \pm 1.1 \mathrm{~g}$, respectively) in 3 weeks of treatment (Fig. 3b). The weight gain/feed ratio was calculated by dividing weight increase by food intake and was expressed as a percentage of control group diet. During the experimental period, the HFD mice showed a ratio of $266.1 \%$, and those given viscothionin at $1 \mathrm{mg} / \mathrm{kg}$ or $10 \mathrm{mg} / \mathrm{kg}$ had ratio of $244.1 \%$ and $172 \%$, respectively (Fig. 3c). During the 3 -week viscothionin treatment period, the weight gain/feed ratio showed a significant decrement in the group given viscothionin at $10 \mathrm{mg} / \mathrm{kg}$ group, as compared with the HFD-fed group (Fig. 3d). Simvastatin $(10 \mathrm{mg} / \mathrm{kg})$ was used for positive control compared with $10 \mathrm{mg} / \mathrm{kg}$ viscothionin. These results indicate a potential anti-obesity effect of viscothionin through the weight gain/feed ratio.

\section{Effects of viscothionin on serum lipid and abdominal visceral fat content}

Three weeks of treatment with $10 \mathrm{mg} / \mathrm{kg}$ viscothionin significantly improved HFD-induced hypercholesterolemia. Serum concentrations of triglycerides and total cholesterol were significantly reduced by the treatment of viscothionin at $10 \mathrm{mg} / \mathrm{kg}$ in the HFD mice (Fig. 4a, b). Serum concentrations of LDL + VLDL cholesterol in mice fed $10 \mathrm{mg} / \mathrm{kg}$ viscothionin were significantly decreased by $36 \%$, compared with the HFD mice (Fig. 4c). Serum concentrations of HDL cholesterol were also changed significantly in mice fed $10 \mathrm{mg} / \mathrm{kg}$ viscothionin as compared with the HFD mice (Fig. $4 \mathrm{~d}$ ).

To confirm abdominal visceral fat content in the obese animal model, we performed micro-CT and produced representative cross-sectional images (Fig. 4e) followed by quantification graph (Fig. 4f). The white area in the abdomen represents the intra-abdominal fat. After 3 weeks of viscothionin treatment, we found a significantly lower abdominal visceral fat content in the mice treated with $10 \mathrm{mg} / \mathrm{kg}$ viscothionin than in the HFD obese mice. These results confirmed that viscothionin exerts an antiobesity effect in HFD-induced obesity animal model. 

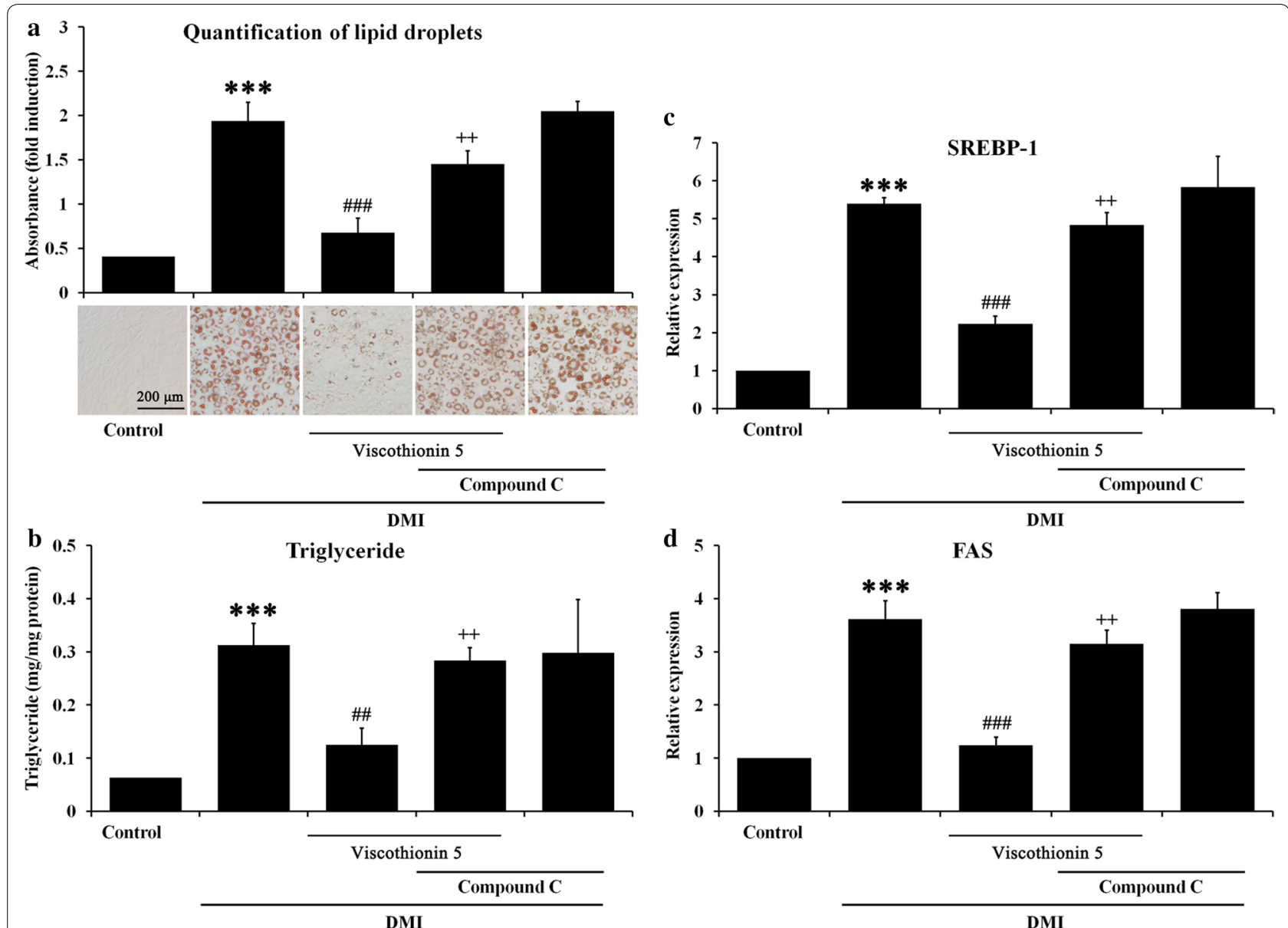

Fig. 2 Lipid accumulation and adipocyte differentiation involved in AMPK activation in viscothionin-treated 3T3-L1 cells. a Cells were pre-treated with compound C ( $20 \mu \mathrm{M})$ for $1 \mathrm{~h}$, then treated with $5 \mu \mathrm{M}$ of viscothionin from days 0 to 8 . On day 8, completely differentiated cells were stained with Oil Red O. Intracellular lipid droplets were stained with Oil Red O and observed at magnification 100x. To quantify lipid accumulation, lipid droplets were eluted with isopropanol and optical absorbance measured at $540 \mathrm{~nm}$. b Compound C counteracted the viscothionin-mediated suppression of the intracellular triglyceride levels of 3T3-L1 cells. c, d SREBP-1 and FAS gene expression in 3T3-L1 cells as examined by real-time RT-PCR. Data are represented as mean \pm SEM of three different preparations. ${ }^{* *} p<0.001$, as compared with control. ${ }^{\# \#} p<0.01$, and ${ }^{\# \#} p<0.001$, as compared with only DMI. ${ }^{++} p<0.01$, as compared with DMI + viscothionin $5 \mu \mathrm{M}$

\section{Discussion}

The present study was conducted to evaluate the antiobesity effects of viscothionin, a potentially protective compound from the traditional herb, Korean mistletoe. Obesity is a major risk factor for metabolic disorders such as diabetes mellitus, hyperlipidemia and hypertension [1]. The body fat distribution has a significant effect on disease morbidity. For example, abdominal adipose tissue decreases insulin sensitivity, while increasing the risk of atherosclerosis and cardiovascular disease [13, 14]. Excess dietary fat itself changes the cholesterol and triglyceride levels in plasma, and the tissues may contribute to obesity and related pathologies [15]. Since body fat is synthesized from mature fat cells through differentiation and maturation of pre-adipocytes, several studies have focused on adipogenic signaling for developing anti-obesity substances [16]. Pre-adipocytes differentiate and mature by answering hormones, including insulin through the expression of adipogenic genes such as SREBP-1 and FAS, and further generates a triglycerides [17]. The pathogenesis of obesity includes failure of hormonal and enzymatic factors, such as hormone-sensitive lipase, low-density lipoprotein receptor, insulin, lipoprotein lipase, and growth hormone [18]. Recently, number of strategies have been proposed as treatments for obesity, including diet and exercise therapy, including chemical drugs for lipid degradation. However, many such anti-obesity treatments exhibit adversary effects due to their ability to alter the control appetite and/or biochemistry of metabolism [19]. 

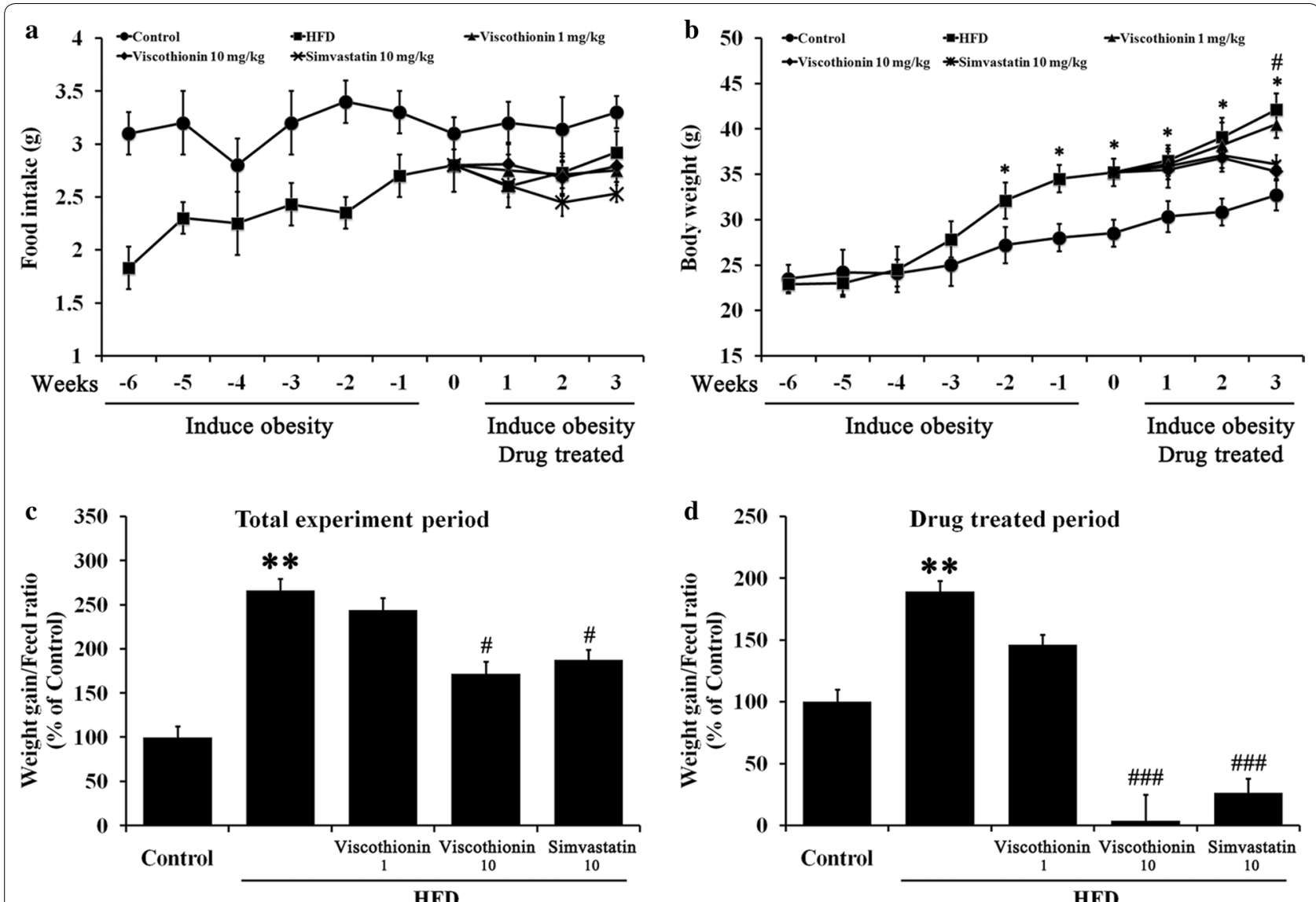

Fig. 3 Effects of viscothionin on food intake and body weight gain of HFD-fed mice. a Effect of viscothionin on change in food intake in HFD mice. Obese mice were induced by HFD for 6 weeks, and then treated with $1 \mathrm{mg} / \mathrm{kg}$ or $10 \mathrm{mg} / \mathrm{kg}$ viscothionin for 3 weeks. $10 \mathrm{mg} / \mathrm{kg}$ of simvastatin was used for comparative control. $\mathbf{b}$ Effect of viscothionin on body weight gain of HFD mice. $\mathbf{c}$ Weight gain/feed ratio during total experiment period. $\mathbf{d}$ Weight gain/feed ratio during viscothionin treatment period. The values are weight gain divided by food intake. The final value in the standard diet group was taken as $100 \%$. ${ }^{*} p<0.05$, and ${ }^{* *} p<0.01$, as compared with control. ${ }^{\#} p<0.05$, and ${ }^{\# \# \#} p<0.001$, as compared with HFD

(See figure on next page.)

Fig. 4 Serum biomarkers and micro-CT findings in viscothionin-treated HFD-fed Mice. Six weeks HFD mice were induced to be obese, and then treated with viscothionin at the dose of $1 \mathrm{mg} / \mathrm{kg}$ or $10 \mathrm{mg} / \mathrm{kg}$ for 3 weeks. $10 \mathrm{mg} / \mathrm{kg}$ of simvastatin was used for comparative control. Serum samples were obtained after viscothionin treatment. $\mathbf{a}$, b Serum concentrations of triglycerides and total cholesterol were measured by a chemistry analyzer. $\mathbf{c}, \mathbf{d} L D L+V L D L$ cholesterol and HDL cholesterol levels were measured with a commercial analysis kit. e Transverse section image of abdominal cavity. White areas represent abdominal visceral fat. $\mathbf{f}$ Quantification of abdominal visceral fat. Transverse imaging measured relative cross-sectional areas of abdominal visceral fat at the mid-kidney level. Note that the area of visceral abdominal fat is lower in the viscothionin-treated mice than in the HFD mice. Values are mean \pm SEM for eight animals in each group. ${ }^{*} p<0.05,{ }^{* *} p<0.01$, and ${ }^{* * *} p<0.001$, as compared with control. ${ }^{*} p<0.05$, and ${ }^{\# \#} p<0.01$, as compared with HFD

Korean mistletoe, a semi-parasitic plant, has been used as an alternative treatment material against several disorders, including cardiac vascular diseases, hypertension, cancer, arthritis, and epilepsy [8-11]. Recently, several studies have shown that Korean mistletoe has possible anticancer [11], and anti-obesity [20] effects. Based on these studies, in our previous research, we have identified a potential candidate molecule, viscothionin, from
Korean mistletoe [12], and herein this study confirmed that viscothionin is an active molecule concerned with lipid metabolism.

In this study, we confirmed the effect of viscothionin on adipocyte differentiation by measuring lipid accumulation and several adipogenic genes and proteins associated with adipogenesis signal pathway. Possible mechanisms for reducing obesity involve decreasing 

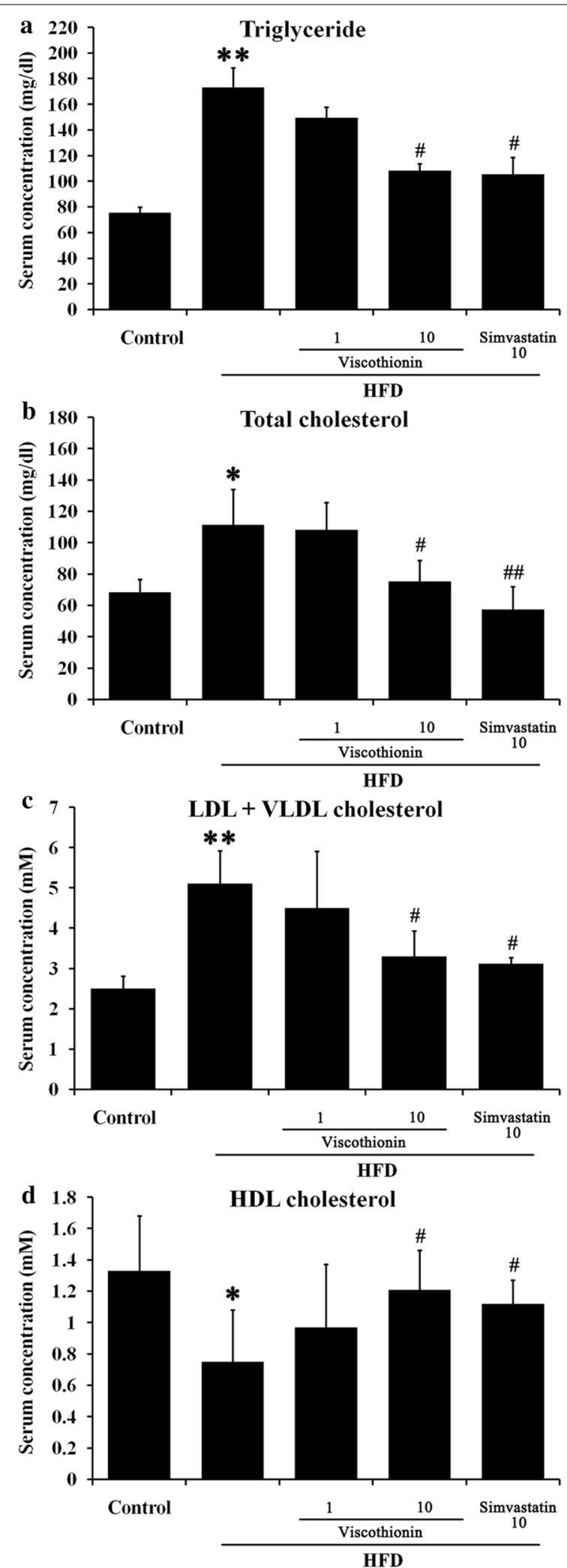

e
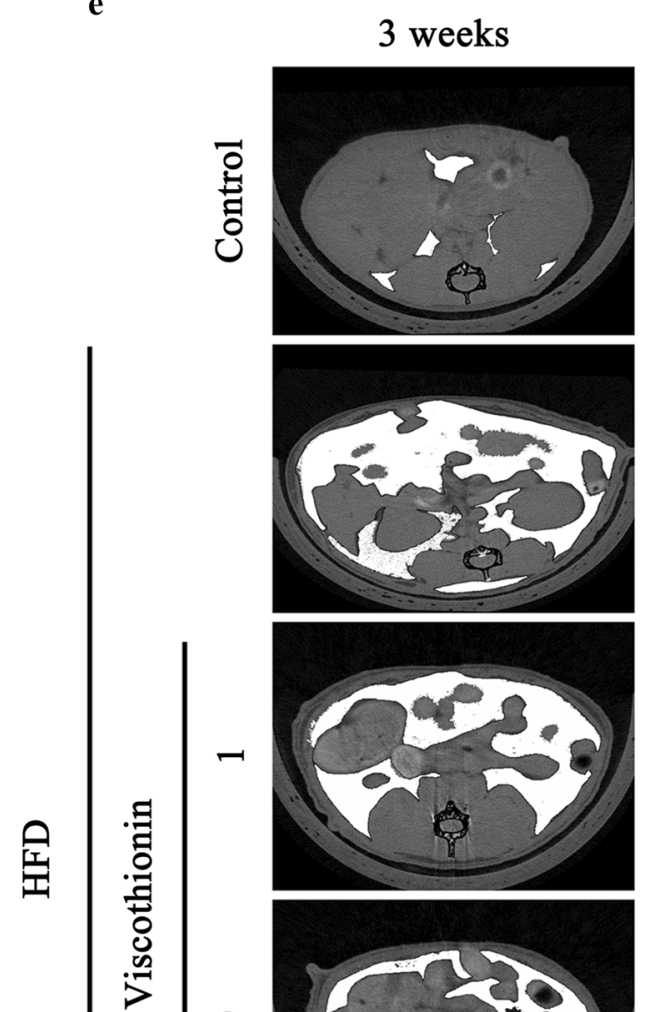

吾

尔
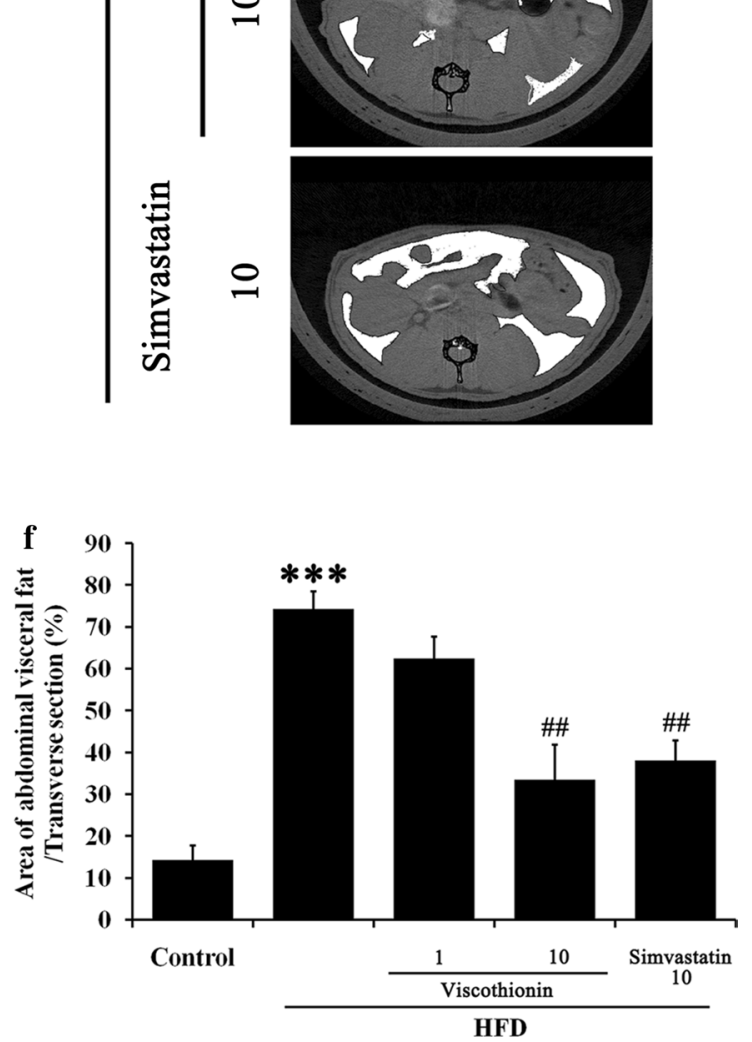
the incorporation of triglycerides and cholesterol, increasing glucose and/or fatty acid oxidation, or increasing lipolysis [21]. Adipocyte differentiation and fat accumulation are known to be associated with the development of obesity [15].

Results of preliminary experimental data indicated that viscothionin inhibited adipocyte differentiation in the 3T3-L1 cells and $5 \mu \mathrm{M}$ concentration of viscothionin is effective at mitigating the accumulation of intracellular lipids. Previous studies have shown that AMPK activation directly inhibits ACC activity through phosphorylation, and indirectly inhibits ACC expression through inhibition of adipogenic signals, including AKT and GSK3 $\beta$ [21]. In the present study, viscothionin increased the phosphorylation of AMPK and ACC, while decreased the phosphorylation of AKT and GSK3 $\beta$ in 3T3-L1 cells (Fig. 1). AMPK cascades have emerged as important targets for the treatment of obesity [22]. Therefore, the anti-adipogenic effect of AMPK is recognized as a major effect of viscothionin in 3T3-L1 cells.

Furthermore, we confirmed the effect of viscothionin on inhibiting obesity in HFD-induced obesity animal model (Fig. 3b). Interestingly, these results were similar to those of mice treated with simvastatin, a commercially available anti-obesity agent. The anti-obesity effect of viscothionin demonstrated that the serum concentrations of LDL + VLDL cholesterol, triglycerides, and total cholesterol were significantly reduced (Fig. 4). Especially the serum concentrations of HDL cholesterol were improved compared with mice treated with simvastatin and were similar to control groups (Fig. 4d).

Additionally, the abdominal visceral fat content was significantly lowered in mice treated with viscothionin than in HFD obese mice (Fig. 4f). Overall, these results demonstrate an anti-obesity effect of viscothionin in an HFD-induced obesity animal model.

\section{Acknowledgements}

This research was supported by "Research Base Construction Fund Support Program" funded by Chonbuk National University in 2019.

\section{Authors' contributions}

SK carried out the experiments, collected the data, and summarized a main finding for the manuscript. SA interpreted a data and drafted the manuscript. $J P, C P$ and YS participated in study design, data evaluation and coordination. GS and JK manage and organize a full manuscript as a corresponding author. All authors read and approved the final manuscript.

\section{Funding}

Not applicable.

\section{Availability of data and materials}

All data used during the current study are available from the corresponding author on reasonable request.

\section{Competing interests}

The authors declare that they have no competing interests.

\section{Author details}

${ }^{1}$ Bio-Safety Research Institute and College of Veterinary Medicine, Chonbuk National University, Iksan 54596, South Korea. ${ }^{2}$ Advanced Radiation Technology Institute, Korea Atomic Energy Research Institute, Jeongeup, Republic of Korea. ${ }^{3}$ Department of Medicinal Crop Research, National Institute of Horticultural and Herbal Science, Rural Development Administration, Eumseong, Republic of Korea.

Received: 13 November 2019 Accepted: 13 January 2020

Published online: 05 February 2020

\section{References}

1. Kopelman PG (2000) Obesity as a medical problem. Nature 404(6778):635-643

2. Ono M, Fujimori K (2011) Antiadipogenic effect of dietary apigenin through activation of AMPK in 3T3-L1 cells. J Agric Food Chem. 59(24):13346-13352

3. Bullo M, Casas-Agustench P, Amigo-Correig P, Aranceta J, Salas-Salvado $J$ (2007) Inflammation, obesity and comorbidities: the role of diet. Public Health Nutr. 10(10a):1164-1172

4. Carling D, Mayer FV, Sanders MJ, Gamblin SJ (2011) AMP-activated protein kinase: nature's energy sensor. Nat Chem Biol. 7(8):512-518

5. Daval M, Foufelle F, Ferre P (2006) Functions of AMP-activated protein kinase in adipose tissue. J Physiol. 574(Pt 1):55-62

6. Gwon SY, Ahn JY, Chung CH, Moon B, Ha TY (2012) Lithospermum erythrorhizon suppresses high-fat diet-induced obesity, and acetylshikonin, a main compound of Lithospermum erythrorhizon, inhibits adipocyte differentiation. J Agric Food Chem. 60(36):9089-9096

7. Chen YY, Lee MH, Hsu CC, Wei CL, Tsai YC (2012) Methyl cinnamate inhibits adipocyte differentiation via activation of the CaMKK2-AMPK pathway in 3T3-L1 preadipocytes. J Agric Food Chem. 60(4):955-963

8. Kienle GS, Kiene H (2010) Review article: Influence of Viscum album L (European mistletoe) extracts on quality of life in cancer patients: a systematic review of controlled clinical studies. Integr Cancer Ther. 9(2):142-157

9. Ostermann T, Bussing A (2012) Retrolective studies on the survival of cancer patients treated with mistletoe extracts: a meta-analysis. Explore. 8(5):277-281

10. Pearl PL, Drillings IM, Conry JA (2011) Herbs in epilepsy: evidence for efficacy, toxicity, and interactions. Semin Pediatr Neurol. 18(3):203-208

11. Khil LY, Kim W, Lyu S, Park WB, Yoon JW, Jun HS (2007) Mechanisms involved in Korean mistletoe lectin-induced apoptosis of cancer cells. World J Gastroenterol. 13(20):2811-2818

12. Kim S, Lee D, Kim JK, Kim JH, Park JH, Lee JW et al (2014) Viscothionin isolated from Korean mistletoe improves nonalcoholic fatty liver disease via the activation of adenosine monophosphate-activated protein kinase. J Agric Food Chem. 62(49):11876-11883

13. Despres JP (1998) The insulin resistance-dyslipidemic syndrome of visceral obesity: effect on patients' risk. Obes Res. 6(Suppl 1):8s-17s

14. Nicklas BJ, Penninx BW, Ryan AS, Berman DM, Lynch NA, Dennis KE (2003) Visceral adipose tissue cutoffs associated with metabolic risk factors for coronary heart disease in women. Diabetes Care 26(5):1413-1420

15. McNamara DJ (2000) Dietary cholesterol and atherosclerosis. Biochim Biophys Acta. 1529(1-3):310-320

16. Chang E, Kim CY (2019) Natural products and obesity: a focus on the regulation of mitotic clonal expansion during adipogenesis. Molecules 24(6):1157

17. Sorisky A (1999) From preadipocyte to adipocyte: differentiation-directed signals of insulin from the cell surface to the nucleus. Crit Rev Clin Lab Sci. 36(1):1-34

18. Hill JO, Peters JC, Wyatt HR (2007) The role of public policy in treating the epidemic of global obesity. Clin Pharmacol Ther. 81(5):772-775

19. Varady KA, Jones PJ (2005) Combination diet and exercise interventions for the treatment of dyslipidemia: an effective preliminary strategy to lower cholesterol levels? J Nutr. 135(8):1829-1835

20. Jung HY, Kim YH, Kim IB, Jeong JS, Lee JH, Do MS et al (2013) The Korean Mistletoe (Viscum album coloratum) Extract Has an Antiobesity Effect and Protects against Hepatic Steatosis in Mice with High-Fat DietInduced Obesity. Evid Based Complement Alternat Med. 2013:168207 
21. Hwang JT, Park IJ, Shin Jl, Lee YK, Lee SK, Baik HW et al (2005) Genistein, EGCG, and capsaicin inhibit adipocyte differentiation process via activating AMP-activated protein kinase. Biochem Biophys Res Commun. 338(2):694-699

22. Fryer LG, Carling D (2005) AMP-activated protein kinase and the metabolic syndrome. Biochem Soc Trans. 33(Pt 2):362-366

\section{Publisher's Note}

Springer Nature remains neutral with regard to jurisdictional claims in published maps and institutional affiliations.
Submit your manuscript to a SpringerOpen ${ }^{\ominus}$ journal and benefit from:

- Convenient online submission

- Rigorous peer review

- Open access: articles freely available online

- High visibility within the field

- Retaining the copyright to your article

Submit your next manuscript at $\boldsymbol{\nabla}$ springeropen.com 